\title{
Low Serum Hepcidin Is Associated With Reduced Short-Term Survival in Adults With Acute Liver Failure
}

\author{
Igor Spivak, ${ }^{1}$ Jyoti Arora, ${ }^{2}$ Caitlyn Meinzer, ${ }^{2}$ Valerie Durkalski-Mauldin, ${ }^{2}$ William M. Lee, ${ }^{3}$ Christian Trautwein, ${ }^{1}$ \\ Robert J. Fontana ${ }^{4}$ Pavel Strnad, ${ }^{1}$ and for the Acute Liver Failure Study Group (ALFSG)
}

\begin{abstract}
The liver has an important role in iron homeostasis through the synthesis of the serum transporter transferrin and the iron hormone hepcidin. The aim of this study was to analyze parameters of iron metabolism in a multicenter cohort of adult patients with acute liver failure (ALF) and in an acetaminophen (APAP)-induced ALF mouse model. A representative subset of 121 adults with ALF (including 66 APAP-related patients) had baseline serum samples tested for ferritin, transferrin, iron, and hepcidin. Outcomes at 3 weeks after enrollment were categorized as spontaneous survivor (SS) versus death/transplantation (NSS). Mice were assessed before (controls) and 4 and 18 hours after injection of $300 \mathrm{mg} / \mathrm{kg}$ APAP. Patients with ALF as well as APAP-treated mice displayed increased ferritin and diminished serum hepcidin and hepcidin/ferritin ratio. SS had lower iron $(29.1 \%$ vs. $34.5 \mu \mathrm{mol} / \mathrm{L}$; $P<0.05)$ and transferrin saturation $(60.9 \%$ vs. $79.1 \% ; P<0.01)$, but higher hepcidin levels $(8.2$ vs. $2.7 \mathrm{ng} / \mathrm{mL}$; $P<0.001)$ and hepcidin/ferritin ratio $(0.0047$ vs. $0.0009 ; P<0.001)$ than NSS. In a multivariate analysis, a log-transformed hepcidin-containing model displayed similar prognostic power as the established Acute Liver Failure Study Group index (C-statistic 0.87 vs. 0.85) and was better than Model for End-Stage Liver Disease score (C-statistic 0.76). In mice, hepcidin levels inversely correlated with the surrogate of liver injury. Conclusion: Our findings demonstrate that several serum iron parameters significantly associate with 3-week outcomes in adults with ALF. Among them, hepcidin decreases early during experimental APAP-induced ALF, is an independent predictor and might be a useful component of future prognostic scores. (Нератоlogy 2019;69:2136-2149).
\end{abstract}

A cute liver failure (ALF) is an uncommon condition characterized by a rapid loss of liver function in individuals without a pre-existing liver disease. ${ }^{(1)}$ In the United States, nearly $50 \%$ of ALF cases are due to acetaminophen (APAP) overdose, whereas idiosyncratic drug-induced liver injury and indeterminate ALF are the most common causes of non-APAP-related ALF and associated with a high rate of short-term mortality. ${ }^{(1)}$ Although liver transplantation is an effective therapy in selected patients, the decision for/ against transplantation is challenging because of the fast pace of disease development, lack of donor organs, and a resulting need for life-long immunosuppressive therapy. ${ }^{(2-4)}$ To facilitate this decision, multiple prognostic markers/scores have been

Abbreviations: ALF, acute liver failure; ALFSG, Acute Liver Failure Study Group; ALT, alanine aminotransferase; APAP, acetaminophen; AST, aspartate aminotransferase; INR, international normalized ratio; MELD, Model for End-Stage Liver Disease; NSS, non-spontaneous survivor; SS, spontaneous survivor; TSAT, transferrin saturation.

Received May 28, 2018; accepted December 10, 2018.

Additional Supporting Information may be found at onlinelibrary.wiley.com/doi/10.1002/hep.30486/suppinfo.

Supported by the German Research Foundation grant STR 1095/4-1, IZKF research group funding, Else Kröner Exzellenzstipendium (to P. S.), SFB/TRR 57 (to P.S. and C.T.), and NIH/NIDDK U-01 58369 to ALFSG.

(C) 2018 by the American Association for the Study of Liver Diseases.

View this article online at wileyonlinelibrary.com.

DOI 10.1002/hep.30486

Potential conflict of interest: Dr. Lee consults for Sanofi, Novartis, Genentech, and Karuna. He received grants from Merck, Gilead, Conatus, Bristol-Myers Squibb, and Synlogic. Dr. Fontana consults for Alnylam and received grants from Bristol-Myers Squibb, Gilead, and AbbVie. 
developed. The Model for End-Stage Liver Disease (MELD) and King's College Criteria are both commonly used prognostic models. ${ }^{(3-5)}$ In addition, the U.S. Acute Liver Failure Study Group (ALFSG) index is a useful prognostic tool ${ }^{(6)}$ that includes the clinical/demographic parameters of ALF etiology, the need for vasopressor use, grade of encephalopathy, and liver-related laboratory values of bilirubin and international normalized ratio (INR). ${ }^{(3,7)}$ Given that the currently available scores have only a limited ability to detect a potentially lethal ALF, there is a constant search for novel predictors. ${ }^{(8-10)}$

Iron is an essential, but potentially toxic, element that causes oxidative stress and promotes the development of bacterial infections. ${ }^{(11-13)}$ Iron is physiologically sequestered as transferrin in the serum and ferritin in the parenchymal cells. ${ }^{(14)}$ Hepatocytes constitute the major parenchymal iron storage pool and contain large amounts of ferritin. A small fraction of ferritin is released into the serum. In the absence of liver injury, serum ferritin is used as a surrogate for the parenchymal iron load. ${ }^{(13-15)}$ The ratio of serum iron and transferrin is termed transferrin saturation (TSAT), indicating the amount of serum iron load. ${ }^{(14)}$ Iron metabolism is regulated by hepcidin, a hormone produced primarily in the hepatocytes that blocks the uptake of iron in the intestine and release of iron from macrophages. ${ }^{(13,15)}$ Hepcidin is a short-living hormone (serum half-life of several minutes) ${ }^{(16)}$ and is subjected to a complex regulation with hypoxia, anemia, and iron deficiency being the major suppressors whereas inflammation and iron overload are the major inducers. $^{(17)}$

Multiple studies demonstrated that parameters of iron metabolism represent useful predictors of liver disease outcome. For example, decreased transferrin and elevated TSAT associated with an adverse outcome in patients with liver cirrhosis, ALF, and acute-on-chronic liver failure. ${ }^{(11,18-20)}$ In contrast, increased ferritin indicated a life-threatening disease in some, but not all, analyses. ${ }^{(11,18,19,21)}$ In studies that measured hepcidin levels, decreased serum hepcidin associated with a poor survival in patients with alcoholic liver cirrhosis. ${ }^{(22)}$ In acute-on-chronic liver failure, the association between low hepcidin and increased mortality was seen in one, but not the other, report. ${ }^{(18,19)}$ Given these data, we aimed to analyze the changes in iron metabolism occurring in ALF and to delineate their prognostic usefulness. For this purpose, we assessed a mouse model of acetaminophen-related ALF and also investigated iron parameters in a multicenter U.S. ALF cohort that were prospectively followed for 3-week outcomes.

\section{Patients and Methods}

\section{ANIMAL EXPERIMENTS}

Ten-to-twelve-week-old wild-type C57BL/6N mice were kept under standardized conditions (12hour day/night cycle, $20^{\circ} \mathrm{C}-24^{\circ} \mathrm{C}$, humidity $50 \%$ ). In the APAP-induced acute liver injury model, mice were fasted for 12 hours before an intraperitoneal injection of $300 \mathrm{mg}$ APAP $/ \mathrm{kg}$ body weight (SigmaAldrich, St. Louis, MO) and received free access to food and water thereafter. ${ }^{(23)}$ The animals were sacrificed 4 or 18 hours after APAP administration by cervical dislocation after an inhalational anesthesia with isoflurane (AbbVie AG, Ludwigshafen, Germany). Control mice of the same age, sex, and genetic background received no treatment. Blood was collected through a cardiac puncture, and serum was obtained through centrifugation for 15 minutes

\section{ARTICLE INFORMATION:}

From the ${ }^{1}$ Medical Clinic III, University Hospital Aachen, Aachen, Germany; ${ }^{2}$ Department of Public Health Sciences, Medical University of South Carolina, Charleston, SC; ${ }^{3}$ UT Southwestern Medical Center at Dallas, Dallas, TX; ${ }^{4}$ University of Michigan, Ann Arbor, MI.

\section{ADDRESS CORRESPONDENCE AND REPRINT REQUESTS TO:}

Pavel Strnad, M.D.

Department of Internal Medicine III and IZKF

University Hospital Aachen

Pauwelsstraße 30
D-52074 Aachen, Germany

E-mail: pstrnad@ukaachen.de

Tel.: +1-49(241) 80-35324 
at $2,000 \mathrm{~g}$. Levels of serum aminotransferases were measured in the Clinical Chemistry Department of Aachen University Hospital. Levels of hepcidin (LSBio LS-F5905; LifeSpan Biosciences, Seattle, WA), ferritin (ab157713; Abcam, Cambridge, United Kingdom), and transferrin (ab157724; Abcam) were measured by commercially available enzyme-linked immunosorbent assay (ELISA) kits as recommended by the suppliers. RNA isolation and complementary DNA preparation were performed with the RNeasy mini kit (Qiagen, Hilden, Germany) and Superscript II reverse transcriptase (Invitrogen, Darmstadt, Germany), respectively. Quantitative PCR was carried out with a 7500 fast Real-Time PCR Sequence Detection System (Applied Biosystems, Foster City, CA). The specific primers are listed in Supporting Table S1. The relative expression levels of mRNA were determined with the help of ddct method and the ribosomal RNA gene L7 was employed as internal control.

Livers were removed, cut, and placed in 10\% buffered formaldehyde overnight. After that, they were dehydrated, embedded in paraffin, and cut into $3-\mu \mathrm{m}$-thick sections. To study the overall tissue architecture, the specimens were stained with hematoxylin and eosin (H\&E). Images were obtained with a Leica light microscope (Leica, Solms, Germany) equipped with a digital camera and Leica Application Suite software V4.1 (Leica Microsystems, Heerbrugg, Switzerland). The animal experiments were approved by the responsible Institutional Animal Care Committee.

\section{PATIENTS}

The analyzed cohort was randomly selected from a pool of 2,244 adult patients that were prospectively identified and recruited at 31 tertiary U.S. centers participating in the ALFSG. ${ }^{(1)}$ All subjects met the ALF criteria, i.e., presence of coagulopathy (international normalized ratio $\geq 1.5$ ) and hepatic encephalopathy occurring within 26 weeks of the first symptoms in individuals without a pre-existing liver disease. ${ }^{(1)}$ The selection of patient samples was carried out by the ALFSG staff not involved in the analysis of obtained results. The institutional review boards of all participating centers approved the research, and the clinical investigation has been conducted according to the principles expressed in the 1975 Declaration of Helsinki. As the patients enrolled had by definition an altered mental status, written informed consent was obtained from the legal next of kin. Monitoring and therapeutic interventions were implemented by each center according to institutional standards of care. Demographic, clinical, laboratory, radiologic, and 21-day transplant-free outcomes data were recorded prospectively.

\section{LABORATORY PARAMETERS}

The measurements of ferritin (electrochemiluminescence; Elecsys ferritin, cat. nr. 04491785 190), transferrin (turbidimetry; Tina-quant Transferrin ver.2), and serum iron (photometry; Iron Gen.2) were performed by the Clinical Chemistry Department of Aachen University Hospital using the Cobas 8000 system (Roche Diagnostics, Mannheim, Germany). The reference ranges were as follows: serum iron 5.8-35 $\mu \mathrm{mol} / \mathrm{L}$; transferrin $200-360 \mathrm{mg} / \mathrm{dL}$; TSAT 25\%-45\%; ferritin 13-150 ng/mL (males), 30-400 $\mathrm{ng} / \mathrm{mL}$ (females). For the determination of serum hepcidin concentrations, a commercially available ELISA kit was used (EIA-5782; DRG Instruments, Marburg, Germany). ${ }^{(19,22,24)}$ Measurements of ferritin, transferrin, iron, and hepcidin provided valid results in 120 (99\%), 121 (100\%), 121 (100\%), and 113 (93\%) patients, respectively. All analyzed samples were obtained from the first two visits after the inclusion of the patient into the study (i.e., day one or two).

\section{STATISTICAL ANALYSIS}

Continuous variables were presented as the mean \pm standard deviation and compared using the Student $t$ test, whereas continuous nonparametric variables were displayed as median (interquartile range) and compared with the Wilcoxon rank-sum test. Categorical variables were presented as number (percentage), and comparison for proportion was done using the chi-squared test or Fisher's exact test. Spearman's correlation coefficient was used to analyze correlations between variables.

The study of associations with transplant-free survival at 21 days after study enrollment was done using a univariate logistic regression model, and all the clinically relevant covariates with $P<0.2$ were chosen for 
a stepwise multivariable logistic regression model. All continuous variables were assessed and the appropriate transformations were done as necessary. All continuous variables were also analyzed for colinearity. The final multivariable model was assessed using the area under the receiver operating characteristic curve (AUROC) or concordance c-statistic - a measure of discriminatory ability. The performance of the final model was compared with two other spontaneous survivor (SS) prediction models based on outcome indices, namely MELD and ALFSG Prognostic Index, using AUROC.

Statistical significance was defined as a two-sided $P$ value $<0.05$. Statistical analyses were performed using SAS software, version 9.4 (SAS Institute Inc., Cary, NC) and RStudio: Integrated Development Environment for R, version 1.1.383 (Rstudio Inc., Boston, MA).

\section{Results}

\section{APAP-INDUCED ACUTE LIVER INJURY IN MICE RESULTS IN ALTERED SERUM IRON PARAMETERS}

The impact of experimental ALF on parameters of iron metabolism was studied in mice subjected to APAP overdose. Eighteen hours after APAP administration, we observed a marked increase in serum aspartate aminotransferase (AST) and alanine aminotransferase (ALT) levels (Supporting Table S2) and the appearance of characteristic centrilobular necrosis in $\mathrm{H} \& \mathrm{E}$ staining (Fig. 1A). Moreover, elevated levels of acute phase response and inflammatory marker genes were noted (Supporting Fig. S1). Although APAP treatment for 18 hours did not result in obvious changes in transferrin (Fig. 1C), it led to significantly elevated serum iron $(38.5 \pm 26.1$ vs. $28 \pm 6.8 \mu \mathrm{mol} / \mathrm{L} ; P<0.01$; Fig. $1 \mathrm{~B})$ and ferritin $(18,830 \pm 33,987$ vs. $871 \pm 384 \mathrm{~g} / \mathrm{L} ; P<0.001$; Fig. 1D) and diminished hepcidin levels $(25.5 \pm 15.3$ vs. $53.3 \pm 24.4 \mathrm{ng} / \mathrm{mL} ; P<0.01$; Fig. 1E). Interestingly, hepcidin levels in APAP-treated mice negatively correlated with ALT $(r=-0.72 ; \quad P<0.01)$ and AST $(r=-0.62 ; P<0.05)$. On the other hand, a significant positive correlation with ALT and AST was seen for ferritin (ALT: $r=0.91$; AST: $r=0.84 ; P<0.0001$ for both; Supporting Table S3). These results suggest that serum ferritin reflects the hepatocellular injury whereas serum hepcidin may mirror the resulting decrease in the synthetic capacity of the liver. In line with this hypothesis, the hepatic mRNA levels of ferritin, transferrin, and hepcidin did not differ significantly between control animals and mice exposed with APAP for 18 hours, thereby demonstrating that the observed serum alterations are not due to transcriptional changes (Supporting Fig. S2).

To test whether the observed changes in serum iron, ferritin, and hepcidin levels occur early after APAP-induced injury, we analyzed mice exposed with APAP for 4 hours. Even at this early time point, the animals displayed a marked elevation in serum AST, ALT, and ferritin levels as well as decreased serum hepcidin values (Supporting Table S4; Supporting Fig. S3). On the other hand, serum iron levels did not differ significantly between the untreated and APAPexposed animals (Supporting Fig. S3).

\section{CHARACTERISTICS OF THE ALF PATIENT COHORT}

To determine the role of iron parameters in human ALF, we analyzed serum samples at the time of enrollment in 121 patients with ALF randomly selected from the prospective multicenter U.S. ALFSG registry (Fig. 2). Our subcohort was representative of the entire registry (Supporting Table S5) and consisted of 66 APAP and 55 non-APAP cases (Fig. 2). In the non-APAP group, autoimmune hepatitis, idiosyncratic drug-induced liver injury, and hepatitis B were the most common etiologies (Supporting Fig. S4). At day 21 after enrollment, 59 subjects (48.8\%) were classified as SS, whereas 30 subjects $(24.8 \%)$ died and 32 subjects (26.4\%) received a liver transplant. The latter two categories were analyzed together and labeled as non-SS (NSS). In line with previous data, ${ }^{(1)}$ spontaneous survival tended to be more frequent in APAPversus non-APAP-induced ALF (57.6\% vs. 38.2\%; $P=0.05$; Fig. 2). Moreover, individuals with APAP etiology were younger and had higher transaminases and higher grades of hepatic encephalopathy, when both admission and peak levels were considered. In contrast, non-APAP ALF cases had higher bilirubin and alkaline phosphatase values and less frequently needed ventilator therapy (Table 1 ). 
A

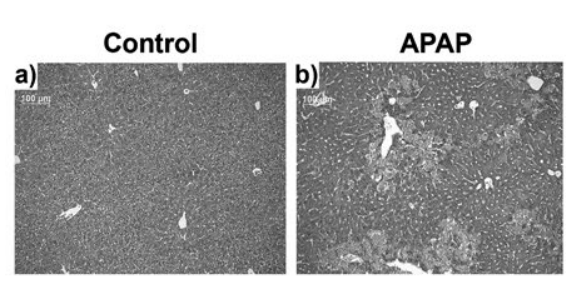

C

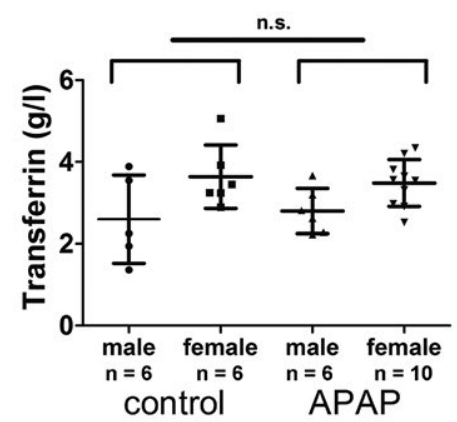

E

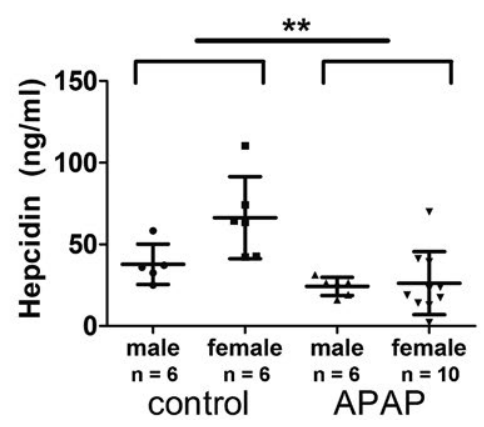

B

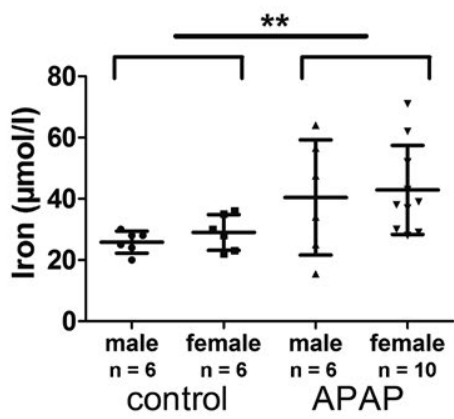

D

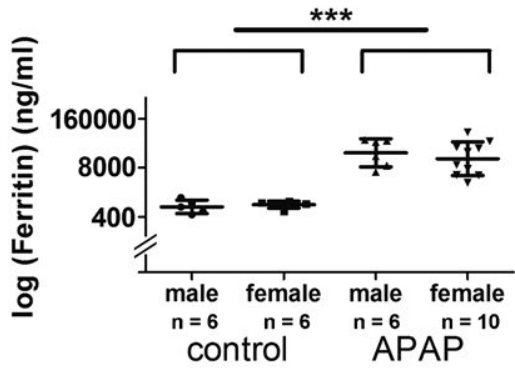

FIG. 1. Acetaminophen-induced liver injury leads to altered parameters of iron metabolism. (A) Hematoxylin and eosin staining reveals the liver architecture in nontreated mice (control; a) and animals exposed to APAP for 18 hours $(\mathrm{b})$. Scale bar $=100 \mu \mathrm{m}$. (B) Serum iron, (C) transferrin, (D), log (ferritin), and (E) hepcidin levels are displayed as means \pm SD. $n$ highlights the amount of analyzed mice. ${ }^{* *}, P<0.01 ;{ }^{* * *}, P<0.001$.

Next, we investigated factors associated with spontaneous survival at day 21 . As reported, ${ }^{(1)}$ females were more likely to display spontaneous survival (84.7\% in SS vs. $64.5 \%$ in NSS; $P<0.05$ ), and SS had lower bilirubin, INR, and MELD scores, when both admission and peak values were considered (Table 2). In contrast, NSS had lower platelet counts and higher grades of hepatic encephalopathy and more frequently needed vasopressor therapy and ventilator support (Table 2).

\section{IRON PARAMETERS ARE} ALTERED IN ALF AND DIFFER BETWEEN SPONTANEOUS SURVIVORS AND NONSPONTANEOUS SURVIVORS

In patients with ALF, serum iron levels were slightly elevated or at the upper limit of normal. ALF individuals also displayed somewhat diminished transferrin values and strongly increased TSAT and ferritin levels 


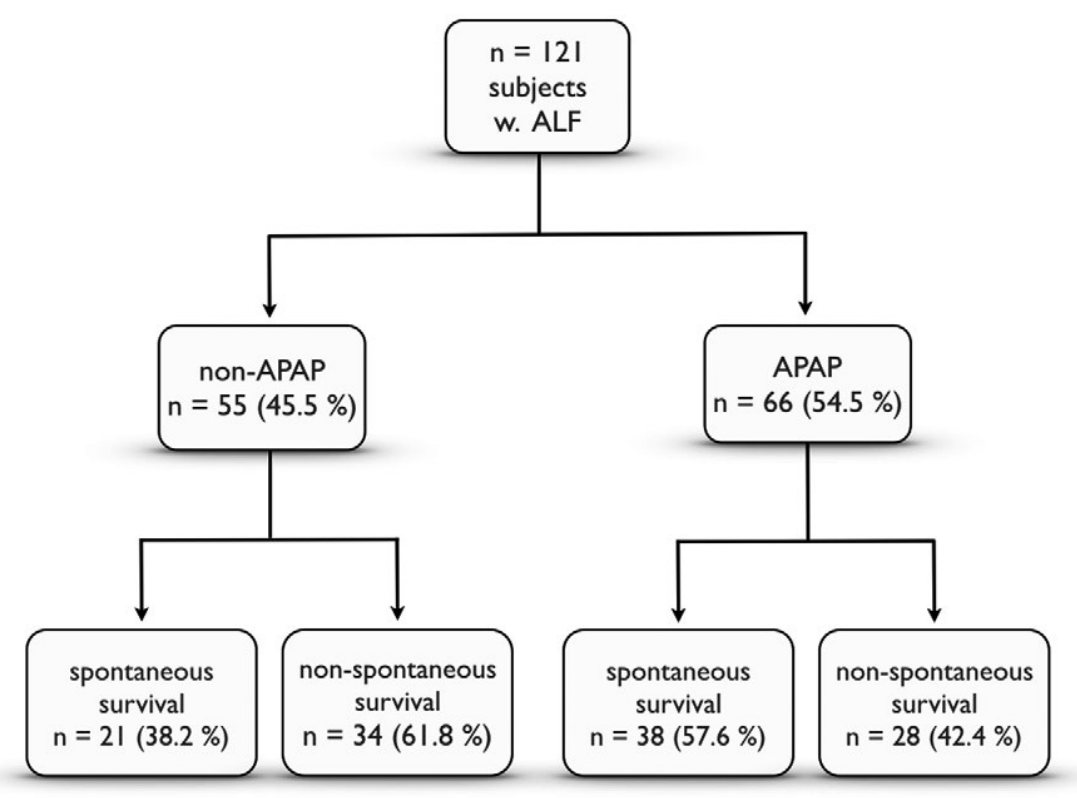

FIG. 2. Overview of the analyzed patients with ALF.

TABLE 1. Comparison of Demographics, Clinical Characteristics, and Iron Results by ALF Etiology

\begin{tabular}{|c|c|c|c|c|}
\hline Variable & $\mathrm{n}$ & Non-APAP $(n=55)$ & $\operatorname{APAP}(n=66)$ & $P$ Value \\
\hline Age (years)* & 121 & $46.0(19.0)$ & $36.0(14.2)$ & $<0.001$ \\
\hline Sex (\% female) & 121 & $35(63.6)$ & $55(83.3)$ & 0.024 \\
\hline Race $(\% \text { Caucasian })^{\dagger}$ & 121 & $35(63.6)$ & $59(89.4)$ & 0.002 \\
\hline Race (\% African American) ${ }^{\dagger}$ & 121 & $16(29.1)$ & $6(9.1)$ & \\
\hline Race $(\% \text { other })^{\dagger}$ & 121 & $4(7.3)$ & $1(1.5)$ & \\
\hline Ethnicity (\% not Hispanic or Latino) ${ }^{\dagger}$ & 121 & $48(87.3)$ & $64(97.0)$ & 0.07 \\
\hline \multicolumn{5}{|l|}{ 21-day outcome } \\
\hline Spontaneous survival (\% yes) & 121 & $21(38.2)$ & $38(57.6)$ & 0.05 \\
\hline \multicolumn{5}{|l|}{ Admission labs } \\
\hline $\operatorname{ALT}(\mathrm{IU} / \mathrm{L})^{*}$ & 119 & $639.0(1,167.0)$ & $3,549.5(4,229.0)$ & $<0.001$ \\
\hline AST (IU/L)* & 120 & $533.5(1,259.5)$ & $4,717.0(6,897.0)$ & $<0.001$ \\
\hline Alkaline phosphate $(\mathrm{IU} / \mathrm{L})^{*}$ & 120 & $172.0(103.8)$ & $120.5(56.0)$ & $<0.001$ \\
\hline Bilirubin $(\mathrm{mg} / \mathrm{dL})^{*}$ & 120 & $18.0(12.3)$ & $4.4(3.6)$ & $<0.001$ \\
\hline Creatinine $(\mathrm{mg} / \mathrm{dL})^{*}$ & 121 & $1.0(1.8)$ & $1.6(1.9)$ & 0.008 \\
\hline Creatinine $^{2}(\mathrm{mg} / \mathrm{dL})^{\ddagger}$ & 121 & $1.1(2.9)$ & $2.1(3.0)$ & 0.02 \\
\hline Hemoglobin $(\mathrm{g} / \mathrm{dL})^{*}$ & 119 & $11.5(3.2)$ & $10.6(2.9)$ & 0.13 \\
\hline INR* & 121 & $2.5(1.4)$ & $3.3(2.7)$ & 0.005 \\
\hline MELD* & 120 & $30.2(13.7)$ & $33.7(8.0)$ & 0.47 \\
\hline Platelet count* & 119 & $134.0(117.5)$ & $122.5(120.8)$ & 0.38 \\
\hline Toxin screen (\% yes) & 95 & $13(33.3)$ & $36(64.3)$ & 0.006 \\
\hline Venous ammonia* & 76 & $79.5(73.2)$ & $103.0(66.2)$ & 0.09 \\
\hline Leucocyte count $\left(\times 10^{9} / \mathrm{L}\right)^{*}$ & 119 & $8.9(5.9)$ & $9.5(9.3)$ & 0.21 \\
\hline \multicolumn{5}{|l|}{ Peak labs } \\
\hline ALT peak (IU/L)* & 121 & $697.0(1,147.5)$ & $4,542.5(4,192.8)$ & $<0.001$ \\
\hline AST peak (IU/L)* & 121 & $566.0(1,285.0)$ & $5,995.0(7,403.9)$ & $<0.001$ \\
\hline
\end{tabular}


TABLE 1. Continued

\begin{tabular}{|c|c|c|c|c|}
\hline Variable & $\mathrm{n}$ & Non-APAP $(n=55)$ & $\operatorname{APAP}(n=66)$ & $P$ Value \\
\hline Bilirubin peak (mg/dL)* & 121 & $21.1(9.9)$ & $7.5(5.9)$ & $<0.001$ \\
\hline Creatinine peak $(\mathrm{mg} / \mathrm{dL})^{*}$ & 121 & $1.7(1.9)$ & $2.5(2.6)$ & 0.015 \\
\hline INR peak* & 121 & $3.0(2.3)$ & $3.9(2.6)$ & 0.11 \\
\hline MELD peak* & 121 & $35.4(13.5)$ & $35.2(9.9)$ & 0.61 \\
\hline Platelet count nadir* & 121 & $99.0(93.0)$ & $70.5(62.5)$ & 0.012 \\
\hline Venous ammonia peak* & 81 & $106.0(84.5)$ & $105.0(71.2)$ & 0.61 \\
\hline \multicolumn{5}{|l|}{ Admission vitals } \\
\hline Diastolic blood pressure $(\mathrm{mm} \mathrm{Hg})^{*}$ & 121 & $68.0(21.0)$ & $67.0(24.5)$ & 0.96 \\
\hline Systolic blood pressure $(\mathrm{mm} \mathrm{Hg})^{*}$ & 121 & $124.0(18.5)$ & $120.0(26.5)$ & 0.28 \\
\hline Weight $(\mathrm{kg})^{*}$ & 117 & $81.5(29.5)$ & $73.0(26.0)$ & 0.06 \\
\hline Body mass index* & 105 & $28.4(7.0)$ & $25.2(7.9)$ & 0.06 \\
\hline \multicolumn{5}{|l|}{ Clinical parameters at admission } \\
\hline HE grade* & 121 & $2.0(2.0)$ & $3.0(2.0)$ & $<0.001$ \\
\hline Coma grade (\% 3/4) & 121 & $16(29.1)$ & $42(63.6)$ & $<0.001$ \\
\hline Pressors (\% yes) & 121 & $6(10.9)$ & $13(19.7)$ & 0.28 \\
\hline RRT (\% Yes) & 121 & $10(18.2)$ & $14(21.2)$ & 0.85 \\
\hline Ventilator (\% yes) & 121 & $15(27.3)$ & $44(66.7)$ & $<0.001$ \\
\hline \multicolumn{5}{|l|}{ Transfusion (before specimen day) } \\
\hline Pre admission FFP (\% yes) & 118 & $20(38.5)$ & $29(43.9)$ & 0.68 \\
\hline Pre admission to specimen day FFP (\% yes) & 121 & $31(56.4)$ & $48(72.7)$ & 0.09 \\
\hline Admission to specimen day RBC (\% yes) & 121 & $16(29.1)$ & $16(24.2)$ & 0.69 \\
\hline Admission to specimen day RVIIA (\% yes) ${ }^{\dagger}$ & 121 & $0(0.0)$ & $3(4.5)$ & 0.25 \\
\hline Admission to specimen day platelets (\% yes) & 121 & $9(16.4)$ & $13(19.7)$ & 0.81 \\
\hline Admission to specimen day Vitamin K (\% yes) & 121 & $4(7.3)$ & $10(15.2)$ & 0.29 \\
\hline Admission to specimen day blood product (\% yes) & 121 & $33(60)$ & $48(72.7)$ & 0.2 \\
\hline \multicolumn{5}{|l|}{ RRT (before specimen day) } \\
\hline Admission to specimen day RRT (\% yes) & 121 & $11(20.0)$ & $16(24.2)$ & 0.74 \\
\hline \multicolumn{5}{|l|}{ Clinical parameters during hospitalization } \\
\hline Peak coma grade during hospitalization* & 121 & $2.0(2.0)$ & $4.0(2.0)$ & 0.009 \\
\hline Pressors during hospitalization (\% yes) & 121 & $14(25.5)$ & $16(24.2)$ & 1 \\
\hline RRT during hospitalization (\% yes) & 121 & $13(23.6)$ & $21(31.8)$ & 0.43 \\
\hline Ventilator during hospitalization (\% yes) & 121 & $22(40.0)$ & $52(78.8)$ & $<0.001$ \\
\hline \multicolumn{5}{|l|}{ Diabetes } \\
\hline Diabetes (\% yes) & 121 & $13(23.6)$ & $10(15.2)$ & 0.34 \\
\hline \multicolumn{5}{|l|}{ Transplant } \\
\hline Ever listed for transplant (\% yes) & 121 & $23(41.8)$ & $25(37.9)$ & 0.8 \\
\hline LTx within 21 days (\% yes) & 121 & $17(30.9)$ & $15(22.7)$ & 0.42 \\
\hline \multicolumn{5}{|l|}{ Iron indices } \\
\hline Ferritin $(\mathrm{ng} / \mathrm{mL})^{*}$ & 120 & $1,935(4,422)$ & $4,179(19,482)$ & 0.018 \\
\hline Iron $(\mu \mathrm{mol} / \mathrm{L})^{*}$ & 121 & $30.6(17.4)$ & $34.5(25.9)$ & 0.08 \\
\hline Transferrin $(\mathrm{mg} / \mathrm{dL})^{*}$ & 121 & $165.0(58.5)$ & $198.0(81.0)$ & 0.008 \\
\hline Hepcidin (ng/mL)* & 113 & $3.3(6.7)$ & $6.8(13.6)$ & 0.008 \\
\hline Transferrin saturation (\%)* & 121 & $71.9(40.7)$ & $71.4(32.3)$ & 0.83 \\
\hline Hepcidin/ferritin ratio* & 112 & $0.0018(0.0063)$ & $0.0016(0.0079)$ & 0.80 \\
\hline
\end{tabular}

Data expressed as median (IQR) for continuous variables and $\mathrm{n}(\%)$ for categorical variables.

*Presented as median (IQR) with Wilcoxon rank-sum $P$ value.

${ }^{\dagger}$ The $P$ value is calculated by the Fisher's exact test.

*If on renal replacement therapy at admission, creatinine set to 4.0.

Abbreviations: FFP, fresh frozen plasma; LTx, liver transplantation; RBC, red blood cells; RRT, renal replacement therapy; rVIIa, recombinant factor VII. 
TABLE 2. Comparison of Demographics and Clinical Characteristics of the Patient Cohort by NSS/SS Status

\begin{tabular}{|c|c|c|c|c|}
\hline Variable & $\mathrm{n}$ & NSS $(n=62)$ & SS $(n=59)$ & $P$ Value \\
\hline Age (years)* & 121 & $40.5(16.8)$ & $38.0(21.0)$ & 0.84 \\
\hline Sex (\% female) & 121 & $40(64.5)$ & $50(84.7)$ & 0.019 \\
\hline Race $(\% \text { Caucasian })^{\dagger}$ & 121 & $48(77.4)$ & $46(78.0)$ & 0.83 \\
\hline Race (\% African American) ${ }^{\dagger}$ & 121 & $12(19.4)$ & $10(16.9)$ & \\
\hline Race $(\% \text { other })^{\dagger}$ & 121 & $2(3.2)$ & $3(5.1)$ & \\
\hline Ethnicity (\% not Hispanic or Latino) ${ }^{\dagger}$ & 121 & $58(93.5)$ & $54(91.5)$ & 0.74 \\
\hline \multicolumn{5}{|l|}{ Admission labs } \\
\hline $\operatorname{ALT}(I U / L)^{*}$ & 119 & $1,629.5(2,846.2)$ & $2,333.0(4,233.0)$ & 0.21 \\
\hline AST (IU/L)* & 120 & $1,524.0(4,350.0)$ & $2,043.0(6,041.5)$ & 0.45 \\
\hline Alkaline phosphate (IU/L)* & 120 & $136.0(83.0)$ & $143.0(96.0)$ & 0.99 \\
\hline Bilirubin (mg/dL)* & 120 & $10.8(14.6)$ & $4.9(10.3)$ & $<0.001$ \\
\hline Creatinine (mg/dL)* & 121 & $1.4(1.8)$ & $1.2(1.7)$ & 0.27 \\
\hline Hemoglobin $(\mathrm{g} / \mathrm{dL})^{*}$ & 119 & $11.2(2.9)$ & $10.7(2.7)$ & 0.56 \\
\hline INR* & 121 & $3.1(2.6)$ & $2.3(2.2)$ & 0.007 \\
\hline MELD score* & 120 & $36.2(11.1)$ & $28.6(8.9)$ & $<0.001$ \\
\hline Platelet count* & 119 & $92.0(102.0)$ & $159.5(119.8)$ & $<0.001$ \\
\hline Venous ammonia $(\mu \mathrm{mol} / \mathrm{L})^{*}$ & 76 & $101.0(80.5)$ & $90.0(48.2)$ & 0.49 \\
\hline Leucocyte count $\left(\times 10^{9} / \mathrm{L}\right)^{*}$ & 119 & $9.6(7.6)$ & $8.8(7.3)$ & 0.47 \\
\hline \multicolumn{5}{|l|}{ Peak labs } \\
\hline ALT peak (IU/L)* & 121 & $1,629.5(3,725.2)$ & $2,471.0(4,108.5)$ & 0.2 \\
\hline AST peak (IU/L)* & 121 & $1,603.5(5,036.0)$ & $2,319.0(6,074.5)$ & 0.38 \\
\hline Bilirubin peak $(\mathrm{mg} / \mathrm{dL})^{*}$ & 121 & $16.8(15.1)$ & $8.5(10.5)$ & $<0.001$ \\
\hline Creatinine peak (mg/dL)* & 121 & $2.3(2.0)$ & $1.6(2.5)$ & 0.15 \\
\hline INR peak* & 121 & $3.9(3.1)$ & $2.8(2.4)$ & $<0.001$ \\
\hline MELD score peak* & 121 & $38.8(8.2)$ & $30.3(8.5)$ & $<0.001$ \\
\hline Platelet count nadir* & 121 & $63.5(53.2)$ & $106.0(85.5)$ & 0.002 \\
\hline Venous ammonia peak $(\mu \mathrm{mol} / \mathrm{L})^{*}$ & 81 & $128.0(90.0)$ & $91.5(70.8)$ & 0.07 \\
\hline \multicolumn{5}{|l|}{ Etiology } \\
\hline APAP (\% yes) & 121 & $28(45.2)$ & $38(64.4)$ & 0.05 \\
\hline \multicolumn{5}{|l|}{ Admission vitals } \\
\hline Diastolic blood pressure (mm Hg)* & 121 & $67.0(24.8)$ & $69.0(22.0)$ & 0.70 \\
\hline Systolic blood pressure $(\mathrm{mm} \mathrm{Hg})^{*}$ & 121 & $120.0(24.2)$ & $124.0(21.0)$ & 0.83 \\
\hline Weight $(\mathrm{kg})^{*}$ & 117 & $76.9(29.0)$ & $77.0(25.5)$ & 0.82 \\
\hline Body mass index* & 105 & $25.9(8.1)$ & $27.3(8.9)$ & 0.55 \\
\hline \multicolumn{5}{|l|}{ Clinical parameters at admission } \\
\hline Hepatic encephalopathy grade* & 121 & $3.0(2.0)$ & $2.0(2.0)$ & 0.002 \\
\hline Coma grade $(\% 3 / 4)$ & 121 & $38(61.3)$ & $20(33.9)$ & 0.005 \\
\hline Vasopressor use (\% yes) & 121 & $16(25.8)$ & $3(5.1)$ & 0.004 \\
\hline RRT (\% yes) & 121 & $17(27.4)$ & $7(11.9)$ & 0.06 \\
\hline Ventilation (\% yes) & 121 & $37(59.7)$ & $22(37.3)$ & 0.023 \\
\hline \multicolumn{5}{|l|}{ Transfusion (before specimen day) } \\
\hline Pre admission FFP (\% yes) & 118 & $32(54.2)$ & $17(28.8)$ & 0.009 \\
\hline Pre admission to specimen day FFP (\% yes) & 121 & $47(75.8)$ & $32(54.2)$ & 0.021 \\
\hline Admission to specimen day RBC (\% yes) & 121 & $25(40.3)$ & $7(11.9)$ & $<0.001$ \\
\hline Admission to specimen day rVIIa (\% yes) ${ }^{\dagger}$ & 121 & $3(4.8)$ & $0(0.0)$ & 0.24 \\
\hline Admission to specimen day platelets (\% yes) & 121 & $17(27.4)$ & $5(8.5)$ & 0.01 \\
\hline Admission to specimen day Vitamin K (\% yes) & 121 & $6(9.7)$ & $8(13.6)$ & 0.70 \\
\hline \multicolumn{5}{|l|}{ RRT (before specimen day) } \\
\hline Admission to specimen day RRT (\% yes) & 121 & $20(32.3)$ & $7(11.9)$ & 0.013 \\
\hline
\end{tabular}


TABLE 2. Continued

\begin{tabular}{|c|c|c|c|c|}
\hline Variable & $\mathrm{n}$ & NSS $(n=62)$ & SS $(n=59)$ & $P$ Value \\
\hline \multicolumn{5}{|l|}{ Clinical parameters during hospitalization } \\
\hline Coma grade during hospitalization* & 121 & $4.0(1.0)$ & $2.0(3.0)$ & $<0.001$ \\
\hline Vasopressor use during hospitalization (\% yes) & 121 & $25(40.3)$ & $5(8.5)$ & $<0.001$ \\
\hline RRT during hospitalization (\% yes) & 121 & $22(35.5)$ & $12(20.3)$ & 0.1 \\
\hline Ventilation during hospitalization (\% yes) & 121 & $48(77.4)$ & $26(44.1)$ & $<0.001$ \\
\hline \multicolumn{5}{|l|}{ Transplant } \\
\hline Ever listed for transplant (\% yes) & 121 & $39(62.9)$ & $9(15.3)$ & $<0.001$ \\
\hline \multicolumn{5}{|l|}{ Iron indices } \\
\hline Ferritin $(\mathrm{ng} / \mathrm{mL})^{*}$ & 120 & $2,755(10,018)$ & $2,925(13,716)$ & 0.58 \\
\hline Iron $(\mu \mathrm{mol} / \mathrm{L})^{*}$ & 121 & $34.5(20.6)$ & $29.1(22.3)$ & 0.016 \\
\hline Transferrin (mg/dL)* & 121 & $172.5(80.2)$ & $186.0(79.5)$ & 0.34 \\
\hline Hepcidin $(\mathrm{ng} / \mathrm{mL})^{*}$ & 113 & $2.7(5.9)$ & $8.2(14.2)$ & $<0.001$ \\
\hline Transferrin saturation (\%)* & 121 & $79.1(21.1)$ & $60.9(47.2)$ & 0.001 \\
\hline Hepcidin/ferritin ratio* & 112 & $0.0009(0.0029)$ & $0.0047(0.0219)$ & $<0.001$ \\
\hline
\end{tabular}

Data expressed as median (IQR) for continuous variables and $\mathrm{n}(\%)$ for categorical variables.

*Presented as median (IQR) with Wilcoxon rank sum $P$ value.

†The $P$ value is calculated by the Fisher's exact test.

Abbreviations: FFP, fresh frozen plasma; RBC, red blood cells; RRT, renal replacement therapy; rVIIa, recombinant factor VII.

(Table 1 and in Materials and Methods). Although the reference range for hepcidin remains to be defined, the detected values tended to be lower than the ones obtained previously in healthy population controls. ${ }^{(24)}$ With regard to ALF etiology, subjects with APAPinduced ALF had higher transferrin (198.0 (81.0) vs. $165.0(58.5) \mathrm{mg} / \mathrm{dL} ; P<0.01)$, hepcidin (6.8 (13.6) vs. $3.3(6.7) \mathrm{ng} / \mathrm{mL} ; P<0.01)$, and ferritin levels $(4,179.0$ $(19,481.7)$ vs. $1,935.0(4,421.8) \mathrm{ng} / \mathrm{mL} ; P<0.05)$ than non-APAP cases (Table 1).

Compared with NSS, SS displayed significantly lower TSAT levels (60.9\% (47.2)\% vs. 79.1\% (21.1)\%; $P<0.01)$ and lower levels of serum iron (29.1 (22.1) vs. $34.5(20.6) \mu \mathrm{mol} / \mathrm{L} ; P<0.05)$, but had higher hepcidin values $(8.2(14.2)$ vs. $2.7(5.9) \mathrm{ng} / \mathrm{mL} ; P<0.001)$ and hepcidin/ferritin ratios $(0.0047(0.0219)$ vs. 0.0009 (0.0029); $P<0.0001$; Table 2). In this sample, ferritin levels were similar among SS and NSS groups $(2,925(13,716)$ vs. $2,755(10,018) \mathrm{ng} / \mathrm{mL} ; P=0.058)$, and no differences in the pattern of association by sex were observed. Although the average serum iron and hepcidin levels differed somewhat between APAP and non-APAP cases (Table 1), the above-described differences between SS and NSS with regard to serum iron, TSAT, hepcidin, and hepcidin/ferritin ratios were concordant in both subgroups (Fig. 3).
Among the iron parameters, hepcidin/ferritin ratio correlated negatively with iron $(r=-0.47 ; P<0.001)$ and TSAT $(r=-0.63 ; P<0.001)$, whereas TSAT and ferritin displayed a positive correlation $(r=0.59$; $P<0.001)$. Hepcidin/ferritin ratio also exhibited a weak negative correlation with parameters of a liver injury/dysfunction such as AST $(r=-0.37 ; P<0.001)$, MELD $(r=-0.32 ; P<0.001)$, and INR $(r=-0.51$; $P<0.001)$, and a similar association was noted between hepcidin and bilirubin $(r=-0.30 ; P<0.001)$. Along the same lines, the ALFSG prognostic index positively correlated with both hepcidin $(r=0.51$, $P<0.001)$ and hepcidin/ferritin ratio $(r=0.33$, $P<0.001$; for details, see Supporting Table S6).

\section{HEPCIDIN CONSTITUTES AN INDEPENDENT PREDICTOR OF 21-DAY TRANSPLANT-FREE SURVIVAL}

To evaluate the prognostic significance of iron parameters for 21-day SS, we performed univariate logistic regression. It revealed a significant predictive value for iron $(P<0.05)$, TSAT $(P<0.01)$, logarithmic transformed (hepcidin $+1 ; P<0.001$ ) and squareroot transformed hepcidin/ferritin ratio $(P<0.001)$ 

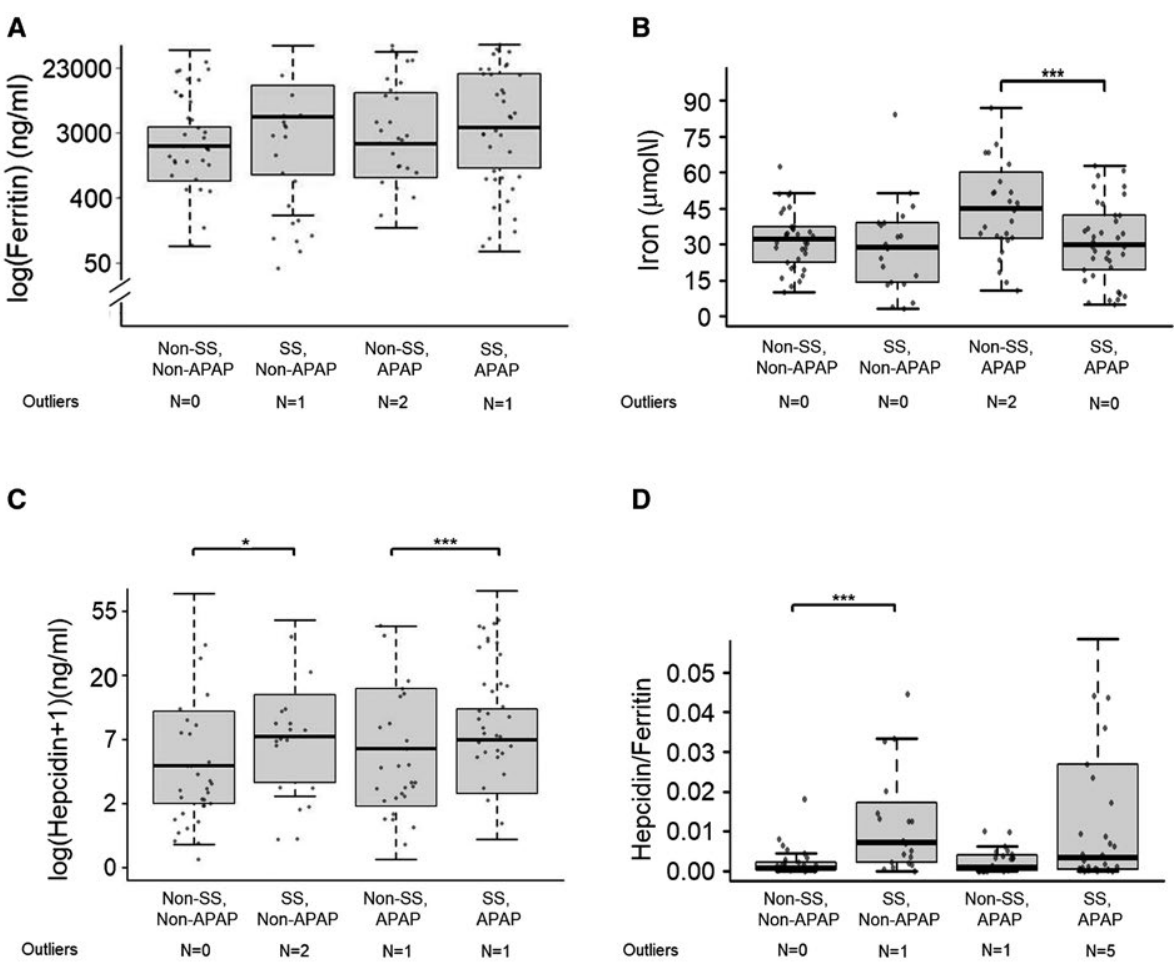

D
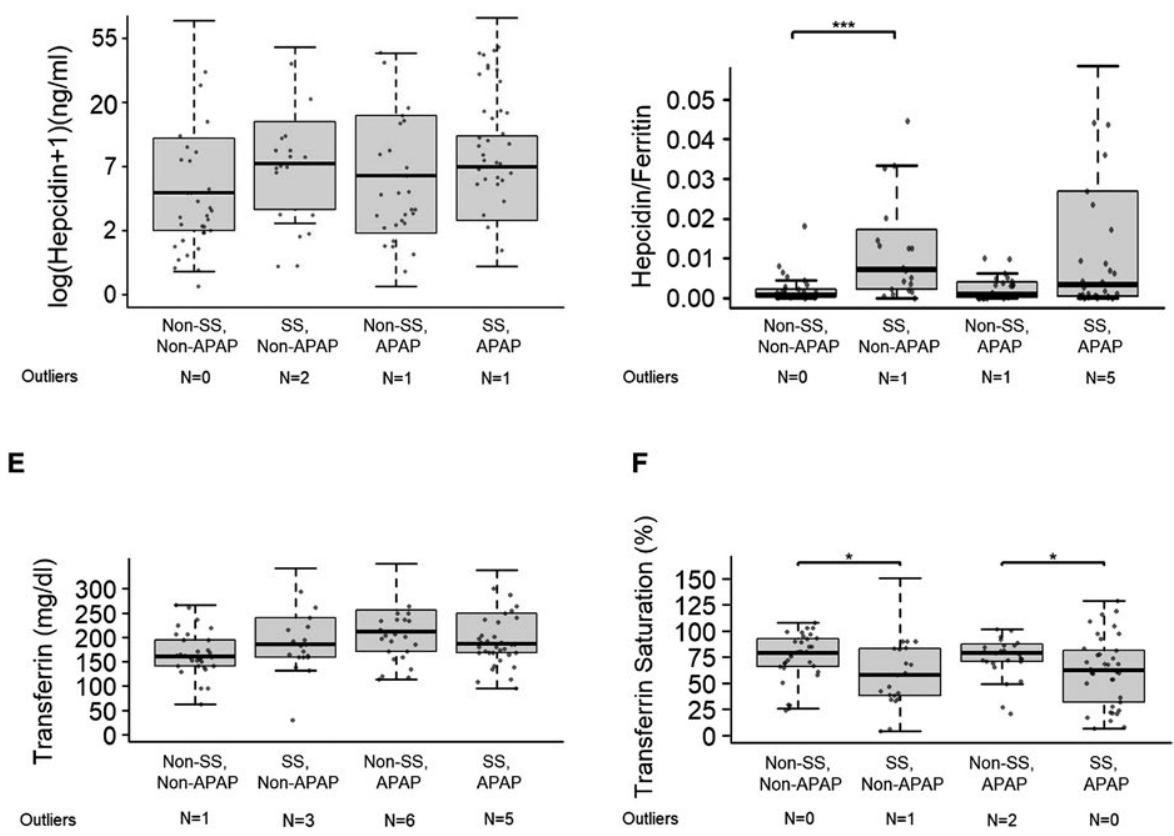

FIG. 3. Parameters of iron metabolism in patients with ALF grouped by etiology and survival status. (A) Log (ferritin), (B) iron, (C) $\log$ (hepcidin+1), (D) hepcidin/ferritin ratio, and (E) transferrin levels as well as (F) TSAT were determined in the highlighted subgroups of patients with ALF. Boxplots display median with first and third quartile, and whiskers indicate smallest and largest nonoutlier observations. Outliers are depicted by empty circles. APAP/non-APAP refers to the ALF etiology (acetaminophen-related or not). Non-SS are individuals who, in contrast with spontaneous survivals (SS), either died or required a liver transplantation within 3 weeks of enrollment. * $P<0.05 ;{ }^{* * *}, P<0.001$.

(Table 3). We also conducted multivariate analysis including all potentially relevant markers. Log (INR; $P<0.05)$, coma grade $3 / 4(P<0.001)$, platelet count $(P<0.001)$, APAP etiology $(P<0.01)$, and $\log$ (hepcidin $+1 ; P<0.05)$ were found as the only parameters that independently associated with 21-day SS (Table 3). To determine the potential usefulness of $\log ($ hepcidin+1) in the clinical routine, we investigated whether its combination with other established markers improves the currently used outcome indices, i.e., the MELD and the ALFSG model. ${ }^{(6)}$ Forwardselection procedures identified a combination of platelet count, log (INR), APAP etiology, coma grade (3/4), and $\log$ (hepcidin+1) as the most parsimonious and best predictive score (Table 4). Compared with both the MELD and ALFSG models, it achieved the highest sensitivity (81\%) and specificity (79.6\%), and the performance of this model was good as defined by the area under the curve (AUC 0.87; 95\% confidence interval [CI] 0.80-0.93) (Table 4, Fig. 4). In contrast, 
TABLE 3. Univariate and Multivariable Logistic Regression Analysis for 21-Day Spontaneous Survival

\begin{tabular}{|c|c|c|c|c|}
\hline \multirow[b]{2}{*}{ Variable } & \multicolumn{2}{|c|}{$\begin{array}{l}\text { Univariate } \\
(\mathrm{n}=121)\end{array}$} & \multicolumn{2}{|c|}{$\begin{array}{c}\text { Multivariate } \\
(\mathrm{n}=121)\end{array}$} \\
\hline & Estimate & $P$ Value & Estimate & $P$ Value \\
\hline Bilirubin (mg/dL) & 0.07015 & 0.002 & & \\
\hline Creatinine $^{2}(\mathrm{mg} / \mathrm{dL})^{*}$ & 0.20672 & 0.07 & & \\
\hline $\log ($ INR) & 1.00832 & 0.009 & 1.4888 & 0.012 \\
\hline MELD & 0.13839 & $<0.001$ & & \\
\hline Platelet count & -0.00797 & 0.002 & -0.0111 & $<0.001$ \\
\hline Height (cm) & 0.03276 & 0.09 & & \\
\hline Iron $(\mu \mathrm{mol} / \mathrm{L})$ & 0.02723 & 0.013 & & \\
\hline Transferrin saturation (\%) & 0.01979 & 0.004 & & \\
\hline Log (hepcidin+1) (ng/mL) & -0.83198 & $<0.001$ & -0.7257 & 0.01 \\
\hline Square root (hepcidin/ferritin) & -14.8873 & $<0.001$ & & \\
\hline APAP (yes) & -0.39361 & 0.035 & -2.2672 & 0.002 \\
\hline Sex (female) & -0.55848 & 0.013 & & \\
\hline Vasopressors (yes) & 0.93522 & 0.005 & & \\
\hline RRT (yes) & 0.51594 & 0.037 & & \\
\hline Ventilation (yes) & 0.45595 & 0.015 & & \\
\hline Coma grade (3/4) & 0.56364 & 0.003 & 2.4221 & $<0.001$ \\
\hline Blood product (yes) & 0.49684 & 0.013 & & \\
\hline Before specimen day RRT (yes) & 0.63170 & 0.009 & & \\
\hline
\end{tabular}

Probability of death or transplant within 21 days is modeled.

*If on renal replacement therapy at admission, creatinine set to 4.0 .

Abbreviation: RRT, renal replacement therapy. the MELD and ALFSG models reached an AUC (95\% CI) of $0.76(0.67-0.85)$ and 0.85 (0.78-0.92), respectively (Table 4; Fig. 4).

\section{Discussion}

Our study demonstrated that patients with ALF display markedly altered iron parameters. However, the observed changes clearly differed from the alterations seen in other liver disorders. In line with a previous report, we saw a massive increase in serum ferritin that surpassed the levels detected in other diseases such as compensated liver cirrhosis, acute-on-chronic liver failure, or sepsis. ${ }^{(11,18,19,21,24)}$ The rise in ferritin was recapitulated in our experimental ALF model and is not surprising because ferritin is present in large amounts in hepatocytes and is released into serum during liver injury. ${ }^{(25)}$ Accordingly, we observed a strong correlation between serum ferritin and the serum liver enzyme levels. Consequently, the high ferritin levels mirror the acute, massive liver damage characteristic for ALF.

Our study and the work from Anastasiou et al. ${ }^{(11)}$ reported very similar levels of serum iron and

TABLE 4. Iron, MELD, and ALFSG Prognosis Model Results

\begin{tabular}{|c|c|c|c|c|c|c|}
\hline & \multicolumn{2}{|c|}{ MELD Model } & \multicolumn{2}{|c|}{ ALFSG Model } & \multicolumn{2}{|c|}{ Iron Model } \\
\hline & OR $(95 \% \mathrm{Cl})$ & $P$ Value & OR (95\% Cl) & $P$ Value & OR $(95 \% \mathrm{Cl})$ & $P$ Value \\
\hline MELD & $1.15(1.1-1.22)$ & $<0.001$ & - & - & - & - \\
\hline Favorable etiology (yes)* & - & - & $0.4(0.08-1.91)$ & 0.25 & - & - \\
\hline APAP etiology (yes) & - & - & - & - & $0.10(0.02-0.45)$ & 0.002 \\
\hline $\log (\mathrm{INR})$ & - & - & $6.96(2.24-21.59)$ & $<0.001$ & $4.43(1.38-14.23)$ & 0.012 \\
\hline Log (bilirubin) & - & - & $3.69(1.6-8.75)$ & 0.003 & - & - \\
\hline Platelet count & - & - & - & - & $0.99(0.98-1)$ & $<0.001$ \\
\hline Log (hepcidin+l) & - & - & - & - & $0.48(0.28-0.84)$ & 0.01 \\
\hline Coma grade (3/4) & - & - & $7.61(2.13-27.21)$ & 0.002 & $11.27(2.91-42.8)$ & $<0.001$ \\
\hline Vasopressors (yes) & - & - & $3.02(0.64-14.3)$ & 0.16 & - & - \\
\hline Sensitivity (\%) & \multicolumn{2}{|c|}{65.6} & \multicolumn{2}{|c|}{73.8} & \multicolumn{2}{|c|}{81.0} \\
\hline Specificity (\%) & \multirow{2}{*}{\multicolumn{2}{|c|}{69.5}} & \multicolumn{2}{|c|}{69.5} & \multicolumn{2}{|c|}{79.6} \\
\hline C-statistic (95\% Cl) & & & \multicolumn{2}{|c|}{$0.85(0.78-0.92)$} & \multicolumn{2}{|c|}{$0.87(0.81-0.94)$} \\
\hline
\end{tabular}

The sensitivity and specificity is based on a cut-point of 0.50 .

*Favorable etiologies: acetaminophen-induced ALF, hepatitis A, pregnancy-related ALF, ischemic liver injury.

Abbreviations: CI, confidence interval; OR, odds ratio. 


\section{ROC Curves}

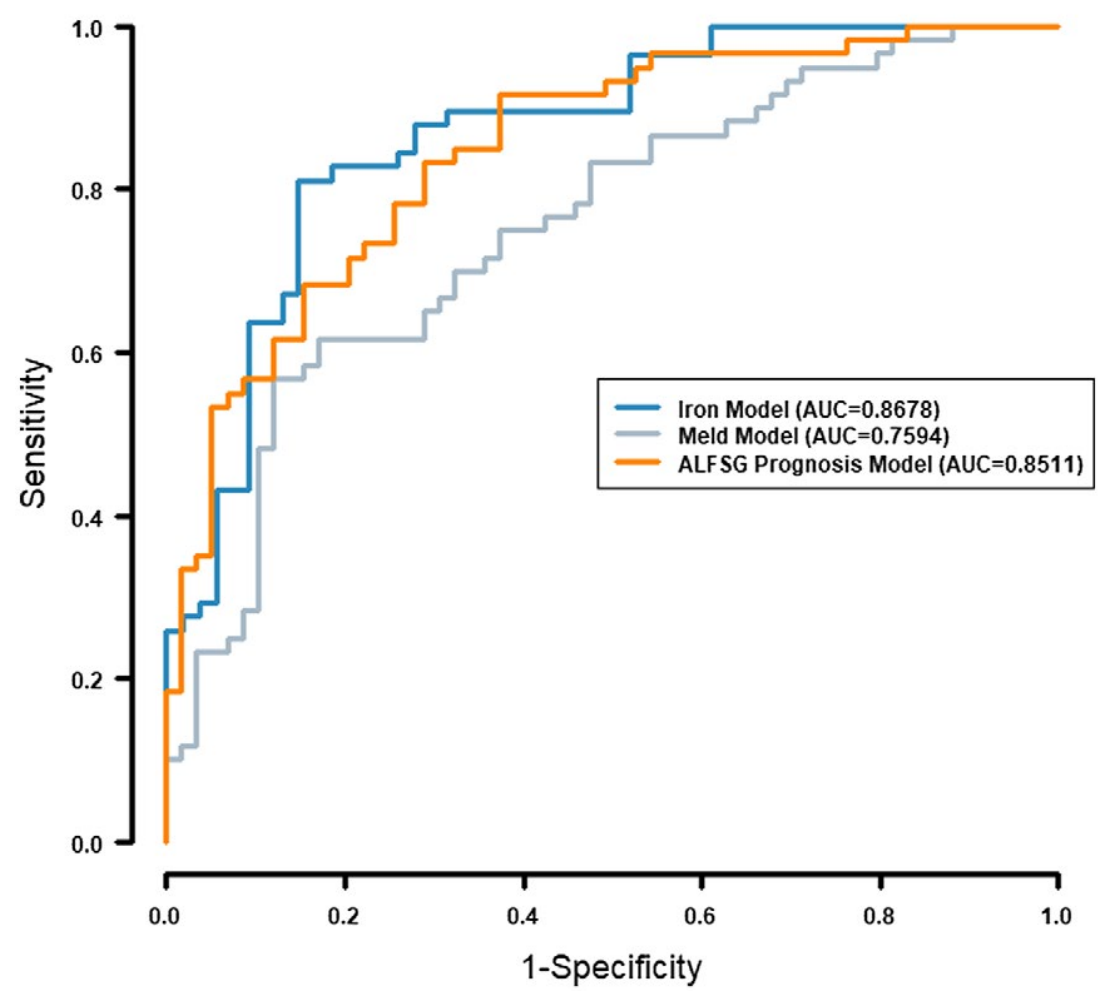

FIG. 4. Diagnostic accuracy of selected models to predict 21-days spontaneous survival in patients with acute liver failure. ROC curves are shown for the MELD, ALFSG (consisting of ALF etiology, need for vasopressor therapy, INR, bilirubin, and coma grade $\geq 3$ ), and iron model (consisting of $\log$ [hepcidin+1], platelet count, log [INR], ALF etiology, and coma grade $\geq 3$ ). AUCs are indicated as $\mathrm{C}$-statistic. Abbreviation: ROC, receiver operating characteristics.

transferrin. Serum iron exceeded the values seen in decompensated liver cirrhosis, acute-on-chronic liver failure, and severe alcoholic hepatitis (unpublished data; also see a previous work $^{(19)}$ ). Moreover, the experimental model demonstrated that the increased serum iron levels constitute a late event during APAP toxicity. These high levels likely reflect the large cell turnover and the diminished hepcidin production. ${ }^{(12)}$ Moreover, the transferrin levels observed in both human studies and the experimental ALF model were above the values detected in individuals with sepsis or decompensated liver cirrhosis. ${ }^{(19,24)}$ These fairly preserved transferrin levels are likely due to the rather long serum half-life of transferrin (8-10 days) ${ }^{(26)}$ and the lack of inflammation that suppresses transferrin production. ${ }^{(24,27)}$

Because of the high serum iron levels, subjects with ALF exhibited TSAT values that were above the ones seen in decompensated cirrhosis or acute-on-chronic liver failure. ${ }^{(18,19,22)}$ These data indicate that ALF leads to a breakdown of the serum iron metabolism. This is not surprising because serum iron represents a very small and dynamic fraction of the total iron body stores that is altered in multiple human disorders. ${ }^{(18,19,24)}$ In particular, the serum iron overload is likely facilitated by decreased iron consumption that is common in critically ill patients ${ }^{(28)}$ as well as by an increased release of iron from damaged cells and red blood cell transfusions.

Even more interestingly, TSAT levels were significantly elevated in NSS individuals compared with survivors. Although a causal impact of elevated TSAT on ALF development remains to be proved, several mechanisms might play a role. First, increased TSAT leads to generation of the highly reactive non-transferrin-bound iron that is known to cause oxidative stress and endothelial damage. ${ }^{(29,30)}$ Moreover, higher iron availability might be exploited by microorganisms 
and thereby predispose to development of microbial infections. ${ }^{(12)}$ Although microbial infection and oxidative stress are of great importance in $\mathrm{ALF}^{(31,32)}$ they also play a crucial role in multiple other disorders. In that respect, high TSAT has been shown to constitute a negative outcome predictor in critically ill patients, individuals with decompensated liver cirrhosis or acute lymphocytic leukemia. ${ }^{(18,19,24,33)}$

Compared with healthy subjects, ${ }^{(24)}$ individuals with ALF displayed decreased hepcidin levels. This is not surprising because diminished hepcidin was reported in patients with impaired liver function. ${ }^{(34,35)}$ In line, our experimental ALF model revealed a strong negative correlation between serum hepcidin levels and the markers of liver injury, and similar, albeit less pronounced, correlation was observed in patients with ALF. In addition to that, hypoxia, the serum iron overload, and oxidative stress are further factors commonly seen in patients with ALF that are known to suppress hepcidin production. ${ }^{(12,34)}$ Although hepcidin is a well-known acute phase reactant, the above-described suppressive factors were obviously more potent than the potential inducers.

Hepcidin levels were particularly low in NSS, and hepcidin constituted an independent predictor of ALF-related survival. These data are reminiscent of the situation in compensated cirrhosis, in which low hepcidin levels were also associated with a poor survival. ${ }^{(22)}$ In contrast, hepcidin did not predict survival in cohorts with a significant amount of microbial infections, including critically ill patients and individuals with acute-on-chronic liver injury. ${ }^{(19,24)}$ Collectively, these observations suggest that in absence of inflammation or in a situation in which other factors outweigh the existing inflammation, hepcidin may serve as a marker of liver function; however, hepcidin also provides added benefits, as demonstrated in the multivariate analysis. Notably, in comparison with the established liver function parameters such as albumin or INR, hepcidin has a much shorter half-life, ${ }^{(36)}$ and the hepcidin changes in the experimental ALF model occurred at an early time point after APAP exposure. Thus, hepcidin might better reflect the dynamic changes occurring in ALF. In contrast, transferrin might be the better predictor in disorders associated with a more pronounced inflammation. ${ }^{(18,19,24)}$

As a possibility to enhance the prognostic usefulness of hepcidin, we analyzed hepcidin/ferritin ratio. Although serum hepcidin and ferritin significantly correlate in healthy subjects, ${ }^{(37)}$ this relationship is altered in advanced liver disease ${ }^{(34)}$ and even more in ALF, which displays low hepcidin but strongly elevated ferritin as a surrogate of the hepatocellular injury (our study; also see previous works ${ }^{(11,25)}$ ). Although hepcidin/ferritin ratio greatly differed between ALF survivors and NSS, multivariate analysis revealed that it may not provide a better predictive value than hepcidin alone.

In conclusion, our study detected unique alterations in iron parameters in individuals with ALF and demonstrated that they may serve as useful predictors of disease outcome. Although they seem to primarily serve as surrogates for the extent of liver injury and decreased liver function, they may also play a causal role in ALF development. Further studies are needed to explore this intriguing possibility as well as to address the usefulness of iron parameters in different ALF etiologies.

\section{REFERENCES}

1) Reuben A, Tillman H, Fontana RJ, Davern T, McGuire B, Stravitz RT, et al. Outcomes in adults with acute liver failure between 1998 and 2013: an observational cohort study. Ann Intern Med 2016;164:724-732.

2) Donnelly MC, Hayes PC, Simpson KJ. The changing face of liver transplantation for acute liver failure: assessment of current status and implications for future practice. Liver Transpl 2016;22:527-535.

3) Wendon J, Cordoba J, Dhawan A, Larsen FS, Manns M, Samuel D, et al. EASL Clinical Practical Guidelines on the Management of Acute (Fulminant) Liver Failure. J Hepatol 2017;66:1047-1081.

4) Flamm SL, Yang YX, Singh S, Falck-Ytter YT, Flamm SL, Lim JK, et al. American Gastroenterological Association Institute Guidelines for the Diagnosis and Management of Acute Liver Failure. Gastroenterology 2017;152:644-647.

5) McPhail MJ, Farne H, Senvar N, Wendon JA, Bernal W. Ability of King's College Criteria and Model for End-Stage Liver Disease scores to predict mortality of patients with acute liver failure: a meta-analysis. Clin Gastroenterol Hepatol 2016;14:516-525.

6) Rutherford A, King LY, Hynan LS, Vedvyas C, Lin W, Lee $\mathrm{WM}$, et al. Development of an accurate index for predicting outcomes of patients with acute liver failure. Gastroenterology 2012;143:1237-1243.

7) Koch DG, Tillman H, Durkalski V, Lee WM, Reuben A. Development of a model to predict transplant-free survival of patients with acute liver failure. Clin Gastroenterol Hepatol 2016;14:1199-1206.

8) Stutchfield BM, Antoine DJ, Mackinnon AC, Gow DJ, Bain CC, Hawley CA, et al. CSF1 restores innate immunity after liver injury in mice and serum levels indicate outcomes of patients with acute liver failure. Gastroenterology 2015;149:1896-1909.

9) Kamath PS, Heimbach J, Wiesner RH. Acute liver failure prognostic scores: is good enough good enough?Clin Gastroenterol Hepatol 2016;14:621-623. 
10) Karvellas CJ, Speiser JL, Tremblay M, Lee WM, Rose CF. Elevated FABP1 serum levels are associated with poorer survival in acetaminophen-induced acute liver failure. HePATology 2017;65:938-949.

11) Anastasiou OE, Kälsch J, Hakmouni M, Kucukoglu O, Heider $\mathrm{D}$, Korth J, et al. Low transferrin and high ferritin concentrations are associated with worse outcome in acute liver failure. Liver Int 2017;37:1032-1041.

12) Ganz $T$, Nemeth E. Iron homeostasis in host defence and inflammation. Nat Rev Immunol 2015;15:500-510.

13) Pietrangelo A. Iron and the liver. Liver Int 2016;36(Suppl 1): 116-123.

14) Camaschella C. Iron-deficiency anemia. N Engl J Med 2015; 372:1832-1843.

15) Rishi G, Subramaniam VN. The liver in regulation of iron homeostasis. Am J Physiol Gastrointest Liver Physiol 2017; 313:157-165.

16) Ruchala $P$, Nemeth $E$. The pathophysiology and pharmacology of hepcidin. Trends in Pharmacol Sci 2014;35:155-161.

17) Sangkhae V, Nemeth E. Regulation of the iron homeostatic hormone hepcidin. Adv Nutr 2017;8:126-136.

18) Maras JS, Maiwall R, Harsha HC, Das S, Hussain S, Kumar C, et al. Dysregulated iron homeostasis is strongly associated with multiorgan failure and early mortality in acute-on-chronic liver failure. Hepatology 2015;61:1306-1320.

19) Bruns $T$, Nuraldeen R, Mai M, Stengel S, Zimmermann HW, Yagmur E, et al. Low serum transferrin correlates with acuteon-chronic organ failure and indicates short-term mortality in decompensated cirrhosis. Liver Int 2017;37:232-241.

20) Viveiros A, Finkenstedt A, Schaefer B, Mandorfer M, Scheiner $\mathrm{B}$, Lehner $\mathrm{K}$, et al. Transferrin as a predictor of survival in cirrhosis. Liver Transpl 2018;24:343-351.

21) Maiwall R, Kumar S, Chaudhary AK, Maras J, Wani Z, Kumar $\mathrm{C}$, et al. Serum ferritin predicts early mortality in patients with decompensated cirrhosis. J Hepatol 2014;61:43-50.

22) Nahon $P$, Nuraldeen $R$, Rufat $P$, Sutton $A$, Trautwein $C$, Strnad P. In alcoholic cirrhosis, low-serum hepcidin levels associate with poor long-term survival. Liver Int 2016;36:185-188.

23) Mossanen JC, Tacke F. Acetaminophen-induced acute liver injury in mice. Lab Anim 2015;49:30-36.

24) Tacke F, Nuraldeen R, Koch A, Strathmann K, Hutschenreuter $\mathrm{G}$, Trautwein C, et al. Iron parameters determine the prognosis of critically ill patients. Crit Care Med 2016;44:1049-1058.

25) Bhagat CI, Fletcher S, Joseph J, Beilby JP. Plasma ferritin in acute hepatocellular damage. Clin Chem 2000;46:885-886.

26) Awai M, Brown EB. Studies of the metabolism of I-131-labeled human transferrin. J Clin Lab Med 1963;61:363-396.
27) Ritchie RF, Palomaki GE, Neveux LM, Navolotskaia O, Ledue TB, Craig WY. Reference distributions for the negative acutephase serum proteins, albumin, transferrin and transthyretin: a practical, simple and clinically relevant approach in a large cohort. J Clin Lab Anal 1999;13:273-279.

28) Darveau M, Denault AY, Blais N, Notebaert E. Bench-tobedside review: iron metabolism in critically ill patients. Crit Care 2004;8:356-362.

29) Brissot $P$, Ropert M, LeLan C, Loréal O. Non-transferrin bound iron: a key role in iron overload and iron toxicity. Biochim Biophys Acta 2012;1820:403-410.

30) Koskenkorva-Frank TS, Weiss G, Koppenol WH, Burckhardt $\mathrm{S}$. The complex interplay of iron metabolism, reactive oxygen species, and reactive nitrogen species: insights into the potential of various iron therapies to induce oxidative and nitrosative stress. Free Radic Biol Med 2013;65:1174-1194.

31) Singal AK, Jampana SC, Weinman SA. Antioxidants as therapeutic agents for liver disease. Liver Int 2011;31:1432-1448.

32) Zider AD, Zopey R, Garg R, Wang X, Wang TS, Deng JC. Prognostic significance of infections in critically ill adult patients with acute liver injury: a retrospective cohort study. Liver Int 2016;36:1143-1150.

33) Potaznik D, Groshen S, Miller D, Bagin R, Bhalla R, Schwartz $\mathrm{M}$, et al. Association of serum iron, serum transferrin saturation, and serum ferritin with survival in acute lymphocytic leukemia. Am J Pediatr Hematol Oncol 1987;9:350-355.

34) Tan TC, Crawford DH, Franklin ME, Jaskowski LA, Macdonald GA, Jonsson JR, et al. The serum hepcidin:ferritin ratio is a potential biomarker for cirrhosis. Liver Int 2012;32:1391-1399.

35) Girelli D, Nemeth E, Swinkels DW. Hepcidin in the diagnosis of iron disorders. Blood 2016;127:2809-2813.

36) Rivera S, Nemeth E, Gabayan V, Lopez MA, Farshidi D, Ganz T. Synthetic hepcidin causes rapid dose-dependent hypoferremia and is concentrated in ferroportin-containing organs. Blood 2005;106:2196-2199.

37) Galesloot TE, Vermeulen SH, Geurts-Moespot AJ, Klaver SM, Kroot JJ, van Tienoven D, et al. Serum hepcidin: reference ranges and biochemical correlates in the general population. Blood 2011;117:218-225.

Author names in bold designate shared co-first authorship.

\section{Supporting Information}

Additional Supporting Information may be found at onlinelibrary.wiley.com/doi/10.1002/hep.30486/suppinfo. 